\title{
A Self-Assembled Iron(II) Metallacage as a Trap for Per- and Poly-fluoroalkyl Substances in Water
}

Cressa Ria P. Fulong, Mary Grace E. Guardian, Diana S. Aga*, Timothy R. Cook*

Department of Chemistry, University at Buffalo, The State University of New York,

Buffalo, New York 14260, United States

Email:dianaaga@buffalo.edu,trcook@buffalo.edu

\section{Supporting Information}

Table of Contents

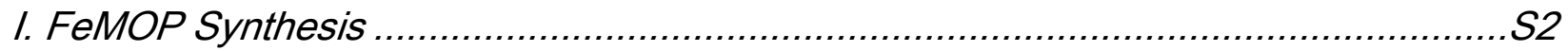

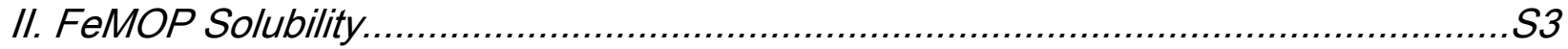

III. Per- and polyfluoroalkyl Substances Chemical Structure ..................................S4

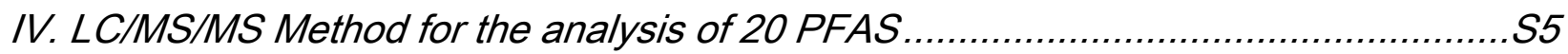

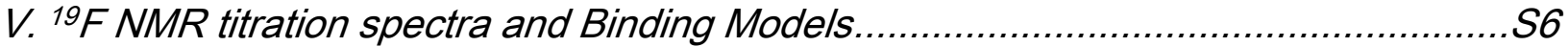

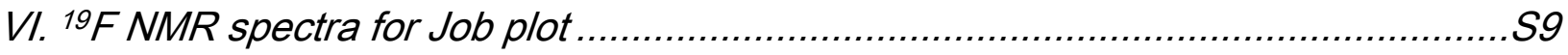




\section{FeMOP Synthesis}

FeMOP ${ }^{1}$ was self-assembled in gram-scale using the following procedure. $5.000 \mathrm{~g}$ (10.15 mmol) 4,4'-diaminobiphenyl-2,2'-disulfonic acid, $2.170 \mathrm{~g}(20.30 \mathrm{mmol})$ 2formylpyridine, $3.685 \mathrm{~g}(20.33 \mathrm{mmol})$ tetramethylammonium hydroxide pentahydrate, and $1.880 \mathrm{~g}(6.750 \mathrm{mmol})$ iron(II) sulfate heptahydrate were added to a $500 \mathrm{~mL}$ Schlenk flask and evacuated for $15 \mathrm{~min}$. Then, $125 \mathrm{~mL}$ degassed deionized $\mathrm{H}_{2} \mathrm{O}$ was added to the flask. The mixture was stirred under $\mathrm{N}_{2}$ for $20 \mathrm{~h}$ at $50^{\circ} \mathrm{C}$. The mixture was then filtered and the product was precipitated by adding $250 \mathrm{~mL}$ acetone. After recrystallization in $500 \mathrm{~mL}$ 1:2 $\left(\mathrm{H}_{2} \mathrm{O}\right.$ :acetone mixture), $5.65 \mathrm{~g}$ of pure product was isolated at quantitative yield. The FTIR spectrum for the synthesized powder is shown in Figure 6. The ${ }^{1} \mathrm{H}$ NMR spectrum is shown in Figure $2 .{ }^{1} \mathrm{H}$ NMR $\left(400 \mathrm{MHz}, \mathrm{D}_{2} \mathrm{O}, 25^{\circ} \mathrm{C}\right): \delta(\mathrm{ppm})=9.34(\mathrm{~s}, 12 \mathrm{H}$, imine $), 8.71$ (d, $12 \mathrm{H},{ }^{3} \mathrm{~J}=7.9 \mathrm{~Hz}, 3$-pyridine), $8.40\left(\mathrm{t}, 12 \mathrm{H},{ }^{3} \mathrm{~J}=8.1 \mathrm{~Hz}, 4\right.$-pyridine), 7.77 (t, 12H, 5- 
pyridine), $7.54\left(\mathrm{~d}, 12 \mathrm{H},{ }^{3} \mathrm{~J}=5.5 \mathrm{~Hz}, 6\right.$-pyridine), $7.14\left(\mathrm{~d}, 12 \mathrm{H},{ }^{3} \mathrm{~J}=8.4 \mathrm{~Hz}, 6,6\right.$ '-benzidine),

$6.44\left(\mathrm{~s}, 12 \mathrm{H}, 3,3^{\prime}\right.$-benzidine), $5.84\left(\mathrm{~d}, 12 \mathrm{H},{ }^{3} \mathrm{~J}=3.6 \mathrm{~Hz}, 5,5^{\prime}\right.$-benzidine), $7.14\left(\mathrm{~s},\left[\mathrm{NMe}_{4}\right]^{+}{ }^{+}\right)$.

FT-IR (ATR, cm-1): 3407 (w), $1640(w), 1608(w), 1582(w), 1553(w), 1491(w), 1471$

(w), $1431(w), 1397(w), 1356(w), 1302(w), 1225(w), 1184(w), 1140(w), 1091(w), 1080$

(w), $1041(w), 1025(w), 997(w), 951(w), 935(w), 906(w), 832(w), 767(w), 750(w)$,

$729(w), 701(w), 672(w), 620(w), 581(w), 550(w)$. 


\section{FeMOP Solubility}
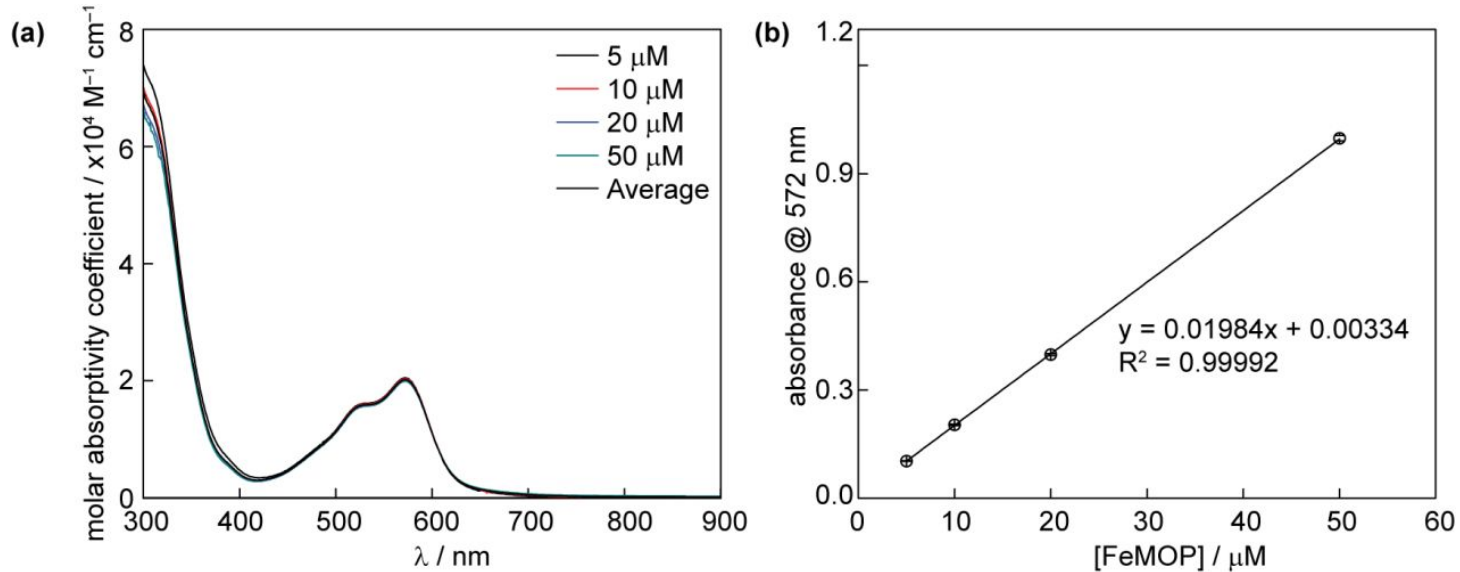

Figure S1. (a) Molar absorptivity coefficient spectra of aqueous FeMOP at 5, 10, 20, and $50 \mu \mathrm{M}$ concentrations and (b) calibration curve using absorbances of FeMOP at $572 \mathrm{~nm}$ to extrapolate the molar solubility. 


\section{Per- and polyfluoroalkyl Substances Chemical Structure}

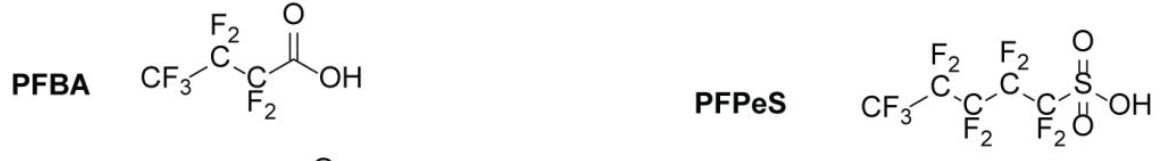

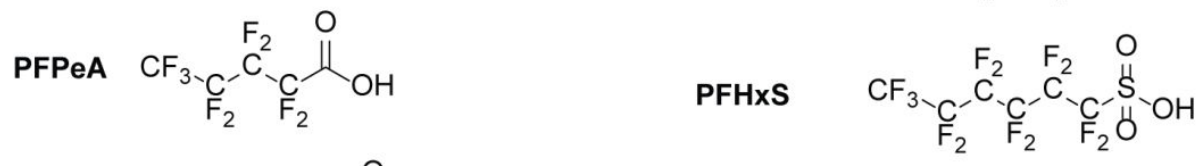

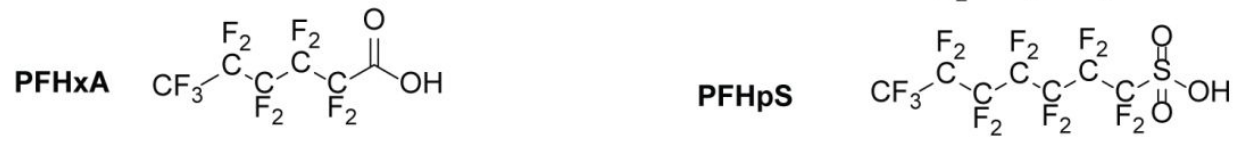

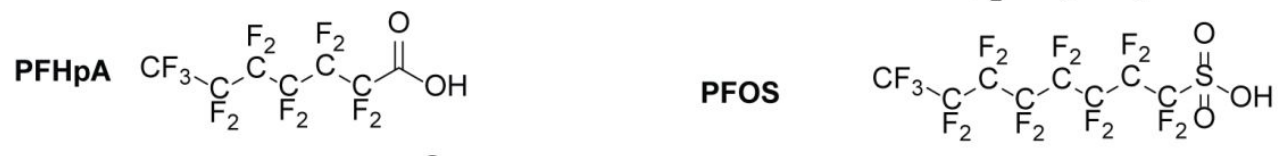

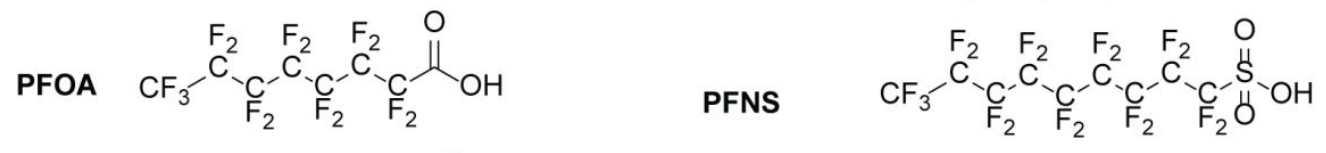

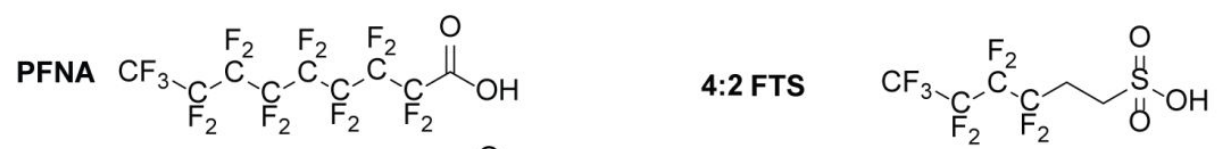

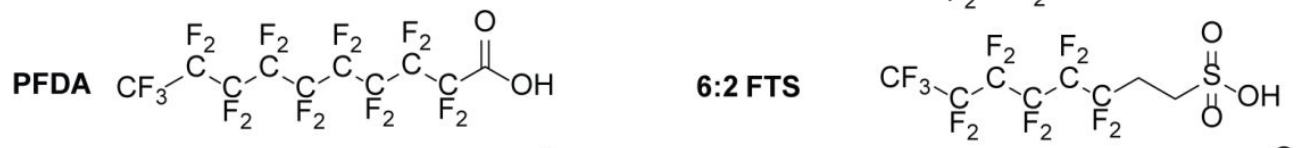

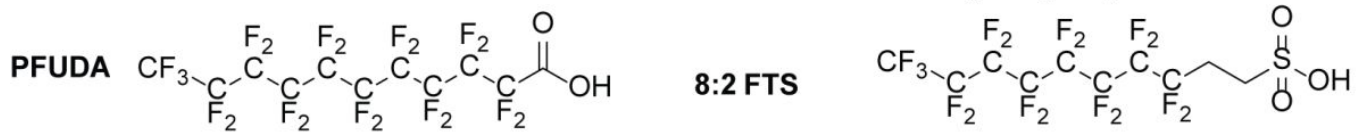

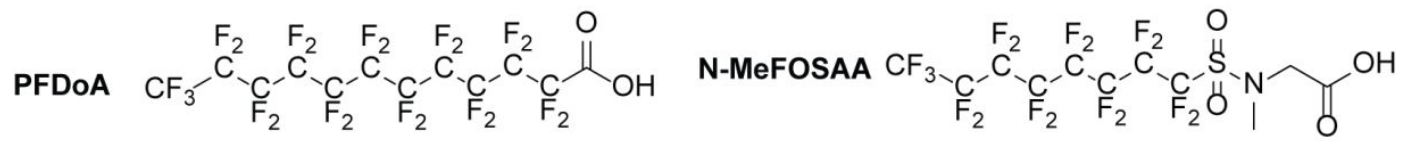

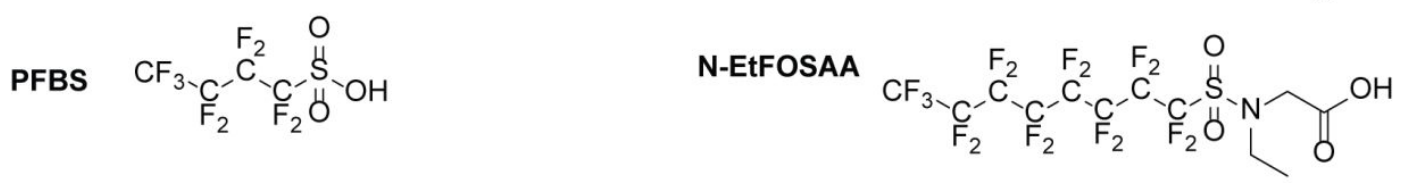

Figure S2. List of per- and polyfluoroalkyl substances (PFASs) included in this work, namely, perfluorobutanoic acid (PFBA), perfluoropentanoic acid (PFPeA), perfluorohexanoic acid (PFHxA), perfluoroheptanoic acid (PFHpA), perfluorooctanoic acid (PFOA), perfluorononanoic acid (PFNA), perfluorodecanoic acid (PFDA), perfluoroundecanoic acid (PFUDA), perfluorododecanoic acid (PFDoA), perfluorobutanesulfonic acid (PFBS), perfluoropentanesulfonic acid (PFPeS), perfluorohexanesulfonic acid (PFHxS), perfluoroheptanesulfonic acid (PFHpS), perfluorooctanesulfonic acid (PFOS), perfluorononanesulfonic acid (PFNS), 4:2 fluorotelomer sulfonate (4:2 FTS), 6:2 fluorotelomer sulfonate (6:2 FTS), 8:2 fluorotelomer 
sulfonate (8:2 FTS), 2-(N-Methylperfluorooctanesulfonamido)acetic acid (N-MeFOSAA), and 2-(N-Ethylperfluorooctanesulfonamido)acetic acid (N-EtFOSAA). 


\section{LC/MS/MS Method for the analysis of 20 PFAS}

A method using a triple quadrupole liquid chromatography with tandem mass spectrometry system [Agilent Technologies HPLC 1200 Series with Thermo Scientific TSQ Quantum Ultra MS] operated under selected reaction monitoring (LC/MS/MS) was optimized for the simultaneous analysis of PFASs compounds. The method uses a Waters X-Bridge ${ }^{\mathrm{TM}} \mathrm{C} 18$ (3.5 $\mu \mathrm{m}$ particle size, $2.1 \mathrm{~mm}$ i.d., $150 \mathrm{~mm}$ length) with a mobile phase consisting of water with $5 \mathrm{mM}$ ammonium acetate (mobile phase A) and acetonitrile (mobile phase B). Gradient elution was carried out at a flow rate of $200 \mu \mathrm{L} \mathrm{min}{ }^{-1}$ starting with $5 \%$ mobile phase $B$, held for 1 min, followed by a 20 -min linear gradient to $90 \%$ mobile phase $\mathrm{B}$, held for $2 \mathrm{~min}$, and then brought back to initial conditions in $1 \mathrm{~min}$ and equilibrated for 10 min before next injection. The sample injection volume was $10 \mu \mathrm{L}$. Summary of the LC/MS/MS parameters for the PFASs included in this study is shown in the table below.

Table S1. LC/MS/MS parameters for the 20 PFASs showing ions monitored [for quantitation (Quan) and for verification (qual)], retention time and instrument linear range. 


\begin{tabular}{|c|c|c|c|c|c|c|c|}
\hline No. & Compound Name & Acronym & $\begin{array}{l}\text { Precursor } \\
\text { lons }\end{array}$ & $\begin{array}{c}\text { Product lons, } \\
\text { Quan/qual }\end{array}$ & $\begin{array}{l}\text { Retention } \\
\text { Time, min }\end{array}$ & $\begin{array}{c}\text { Instrument } \\
\text { Linear } \\
\text { Range, } \\
\mu \mathrm{g} \mathrm{L}^{-1}\end{array}$ & $\begin{array}{l}\text { Linear } \\
\text { Correlation, } \\
\mathbf{R}^{2}\end{array}$ \\
\hline 1 & Perfluorobutanoic acid & PFBA & 213 & $169 / 115$ & 8.9 & $3-250$ & 0.993 \\
\hline 2 & Perfluoropentanoic acid & PFPeA & 263 & $219 / 187$ & 9.5 & $3-250$ & 0.997 \\
\hline 3 & Perfluorohexanoic acid & PFHxA & 313 & $269 / 297$ & 9.8 & $3-150$ & 0.994 \\
\hline 4 & Perfluoroheptanoic acid & PFHpA & 363 & $319 / 169$ & 10.2 & $3-150$ & 0.991 \\
\hline 5 & Perfluorooctanoic acid & PFOA & 413 & $369 / 169$ & 10.5 & $3-150$ & 0.992 \\
\hline 6 & Perfluorononanoic acid & PFNA & 463 & $419 / 219$ & 10.9 & $3-150$ & 0.997 \\
\hline 7 & Perfluorodecanoic acid & PFDA & 513 & $469 / 269$ & 11.3 & $3-150$ & 0.995 \\
\hline 8 & Perfluoroundecanoic acid & PFUdA & 563 & $519 / 269$ & 11.9 & $3-150$ & 0.999 \\
\hline 9 & Perfluorododecanoic acid & PFDoA & 613 & $569 / 319$ & 12.5 & $3-150$ & 0.998 \\
\hline 10 & Perfluorobutanesulfonic acid & PFBS & 299 & $80 / 99$ & 10.1 & $5-150$ & 0.992 \\
\hline 11 & Perfluoropentanesulfonic acid & PFPeS & 349 & $80 / 99$ & 10.4 & $5-150$ & 0.994 \\
\hline 12 & Perfluorohexanesulfonic acid & PFHxS & 399 & $80 / 99$ & 10.8 & $5-150$ & 0.996 \\
\hline 13 & Perfluoroheptanesulfonic acid & PFHpS & 449 & $80 / 99$ & 11.2 & $5-150$ & 0.993 \\
\hline 14 & Perfluorooctanesulfonic acid & PFOS & 499 & $80 / 99$ & 11.7 & $5-150$ & 0.999 \\
\hline 15 & Perfluorononanesulfonic acid & PFNS & 549 & $80 / 99$ & 12.1 & $5-150$ & 0.999 \\
\hline 16 & $\begin{array}{c}2-(N- \\
\text { Methylperfluorooctanesulfonamido)acetic } \\
\text { acid }\end{array}$ & $\begin{array}{c}\mathrm{N}- \\
\text { MeFOSAA }\end{array}$ & 570 & $419 / 483$ & 11.3 & $5-250$ & 0.992 \\
\hline 17 & $\begin{array}{l}\text { Ethylperfluorooctanesulfonamido)acetic } \\
\text { acid }\end{array}$ & $\begin{array}{c}\mathrm{N}- \\
\text { EtFOSAA }\end{array}$ & 584 & $419 / 526$ & 11.5 & $5-250$ & 0.999 \\
\hline 18 & Fluorotelomer sulfonic acid $4: 2$ & $4: 2$ FTS & 327 & $307 / 80$ & 9.7 & $5-250$ & 0.994 \\
\hline 19 & Fluorotelomer sulfonic acid 6:2 & $6: 2$ FTS & 427 & $407 / 80$ & 10.3 & $3-250$ & 0.995 \\
\hline 20 & Fluorotelomer sulfonic acid 8:2 & 8:2 FTS & 527 & $507 / 80$ & 11.1 & $3-250$ & 0.992 \\
\hline
\end{tabular}




\section{V. ${ }^{19} \mathrm{~F}$ NMR titration spectra and Binding Models}

(a)

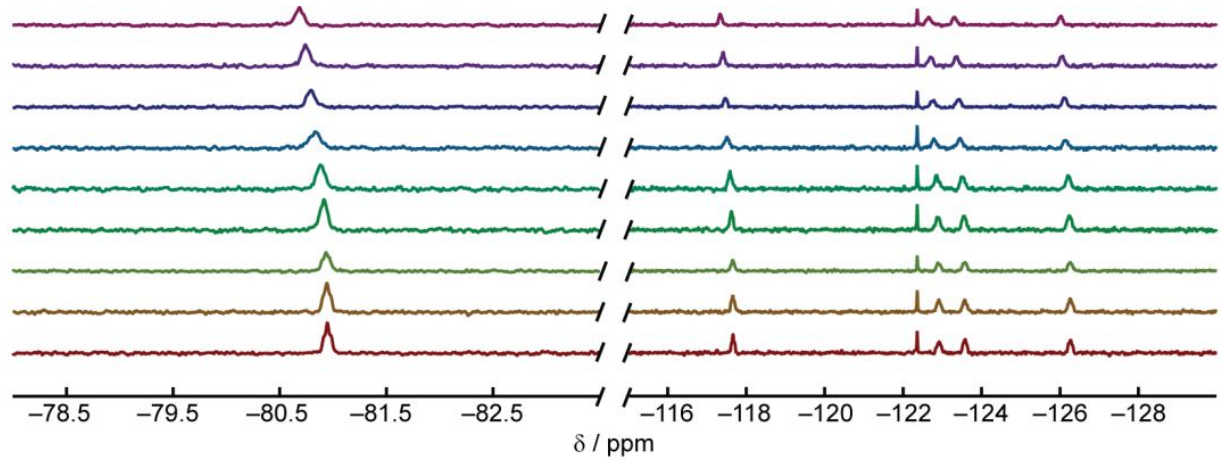

(b)

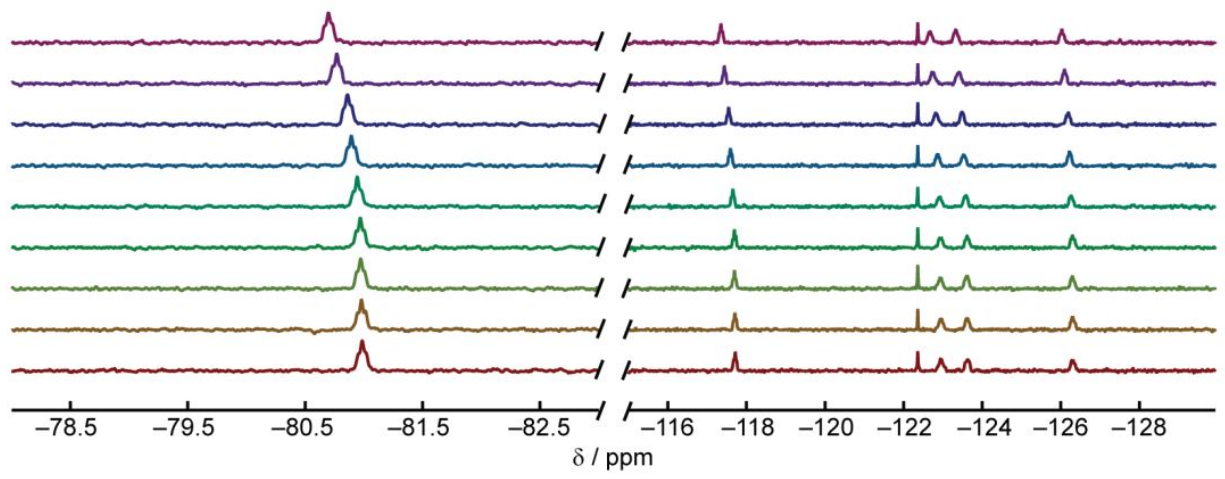

— 0:1 (FeMOP:PFHXA)

- 1:10 (FeMOP:PFHxA)

- 1:4 (FeMOP:PFHXA)

- 1:2 (FeMOP:PFHxA)

- 1:1 (FeMOP:PFHXA)

- 2:1 (FeMOP:PFHxA)

- 3:1 (FeMOP:PFHXA)

- 4:1 (FeMOP:PFHXA)

- 5:1 (FeMOP:PFHXA)

Figure S3. ${ }^{19} \mathrm{~F}$ NMR spectra of $0.5 \mathrm{~mL} \mathrm{D} \mathrm{D}_{2} \mathrm{O}$ solution titration of (a) FeMOP and (b) $\mathrm{FeSO}_{4}$ in $1.0 \mathrm{mM}$ PFHxA referenced at $-122.36 \mathrm{ppm}$ using $5.00 \mathrm{mM} \mathrm{NaF}$ solution in $\mathrm{D}_{2} \mathrm{O}$. 
(a)

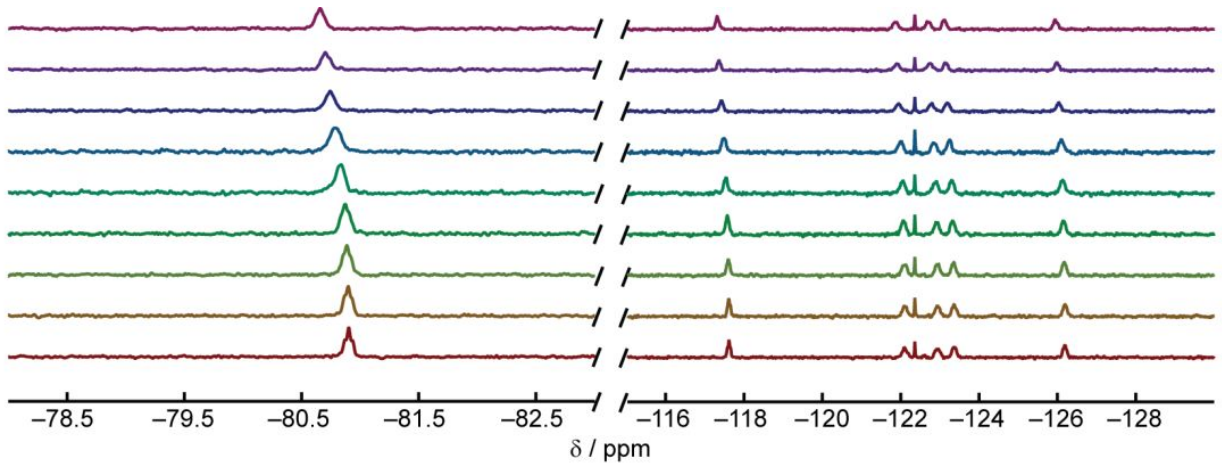

(b)

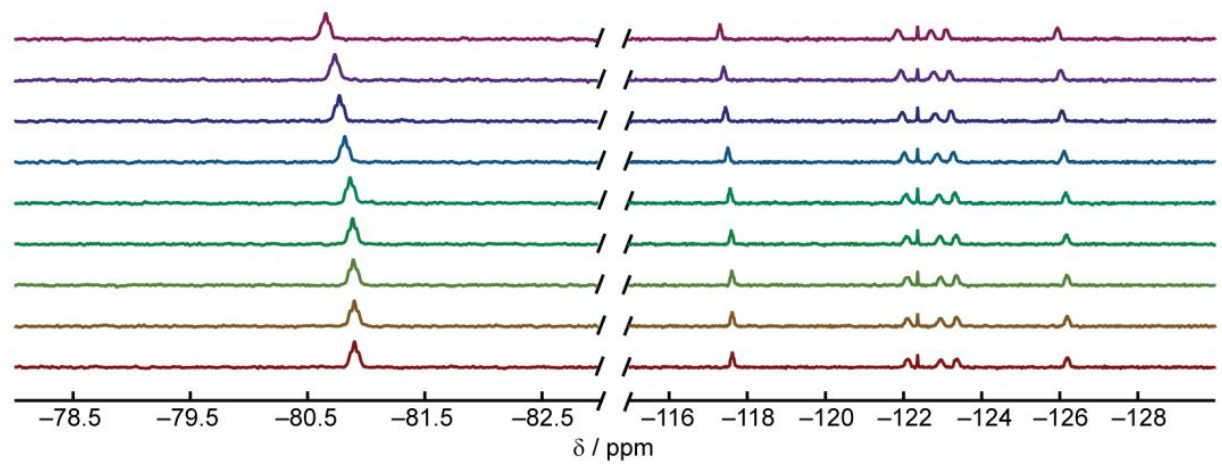

— 0:1 (FeMOP:PFHpA) - 1:10 (FeMOP:PFHpA)

- 1:4 (FeMOP:PFHpA)

- 1:2 (FeMOP:PFHpA)

— 1:1 (FeMOP:PFHpA)

- 2:1 (FeMOP:PFHpA)

- 3:1 (FeMOP:PFHpA)

- 4:1 (FeMOP:PFHpA)

- 5:1 (FeMOP:PFHpA)

— 0:1 ( $\left.\mathrm{FeSO}_{4}: \mathrm{PFHpA}\right)$

- 1:10 ( $\left.\mathrm{FeSO}_{4}: \mathrm{PFHpA}\right)$

— 1:4 ( $\left.\mathrm{FeSO}_{4}: \mathrm{PFHpA}\right)$

- 1:2(FeSO $:$ PFHpA)

- 1:1 ( $\left.\mathrm{FeSO}_{4}: \mathrm{PFHpA}\right)$

- 2:1 (FeSO $:$ PFHpA)

- 3:1 (FeSO $:$ PFHpA)

- 4:1 ( $\left.\mathrm{FeSO}_{4}: \mathrm{PFHpA}\right)$

- 5:1 ( $\left.\mathrm{FeSO}_{4}: \mathrm{PFHpA}\right)$

Figure S4. ${ }^{19} \mathrm{~F}$ NMR spectra of $0.5 \mathrm{~mL} \mathrm{D} \mathrm{D}_{2} \mathrm{O}$ solution titration of (a) FeMOP and (b) $\mathrm{FeSO}_{4}$ in $1.0 \mathrm{mM}$ PFHpA referenced at $-122.36 \mathrm{ppm}$ using $5.00 \mathrm{mM} \mathrm{NaF}$ solution in $\mathrm{D}_{2} \mathrm{O}$. 
Table S2. Summary of remaining PFHpA concentration in solution after addition of FeMOP

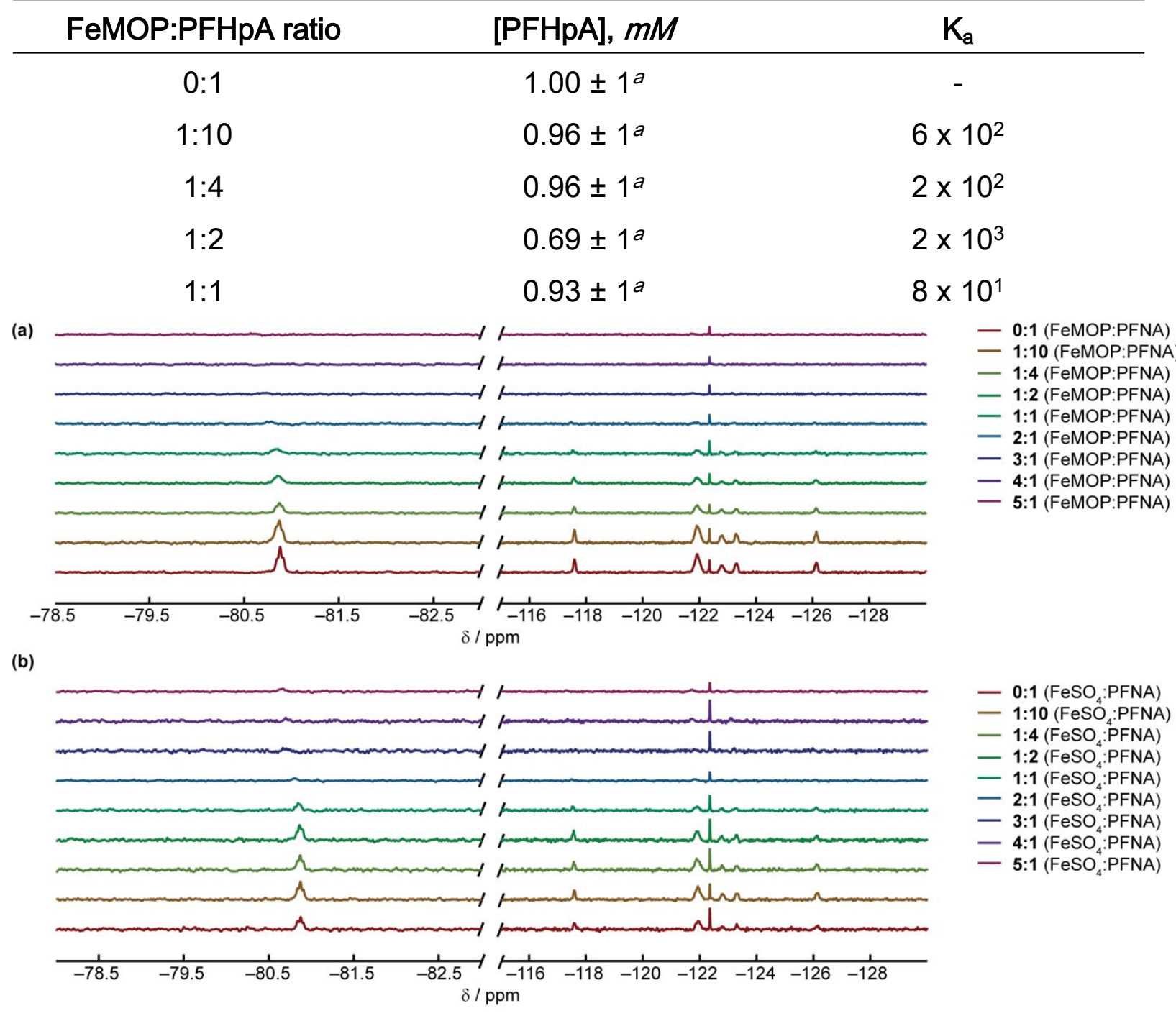

Figure S5. ${ }^{19} \mathrm{~F}$ NMR spectra of $0.5 \mathrm{~mL} \mathrm{D} \mathrm{D}_{2} \mathrm{O}$ solution titration of (a) FeMOP and (b) $\mathrm{FeSO}_{4}$ in $1.0 \mathrm{mM}$ PFNA referenced at -122.36 ppm using $5.00 \mathrm{mM} \mathrm{NaF}$ solution in $\mathrm{D}_{2} \mathrm{O}$. 
Table S3. Summary of fit parameters for the titration binding modeling of FeMOP and $\mathrm{FeSO}_{4}$ association with PFHxA, PFHpA, and PFNA

\begin{tabular}{|c|c|c|c|c|c|c|}
\hline Complex & Model & $K_{a 1}$ & $K_{a 2}$ & $\begin{array}{l}\text { Sum of } \\
\text { squares }\end{array}$ & $\begin{array}{c}\text { Standard } \\
\text { Error }\end{array}$ & $\begin{array}{c}\text { Covariance } \\
\text { of fit }\end{array}$ \\
\hline & 1.1 & $28.01 \pm$ & & 0.00115 & & 0.0032529 \\
\hline FeMOP:PFHx & 1.1 & $63 \%^{a}$ & - & 6 & 0.006107 & 37 \\
\hline \multirow[t]{3}{*}{$A$} & 1.? & & & 0.00110 & & 0.0030943 \\
\hline & 1.2 & $26.43^{b}$ & $-14.17^{c}$ & 1 & 0.006508 & 82 \\
\hline & 1.1 & & & 0.00104 & & 0.0033382 \\
\hline FeMOP:PFHp & 1.1 & $37.52^{b}$ & - & 5 & 0.005805 & 04 \\
\hline \multirow[t]{2}{*}{ A } & $1 \cdot 2$ & $28.87 \pm$ & & 0.00102 & & 0.0032571 \\
\hline & 1.2 & $50 \%^{a}$ & $31.24^{b}$ & 0 & 0.006262 & 88 \\
\hline & $1 \cdot 1$ & & & 0.00305 & & 0.0878690 \\
\hline FDMOD.PFN $\triangle$ & 1.1 & $606.25^{b}$ & - & 6 & 0.012683 & 86 \\
\hline
\end{tabular}




\section{VI. ${ }^{19} \mathrm{~F}$ NMR spectra for Job plot}

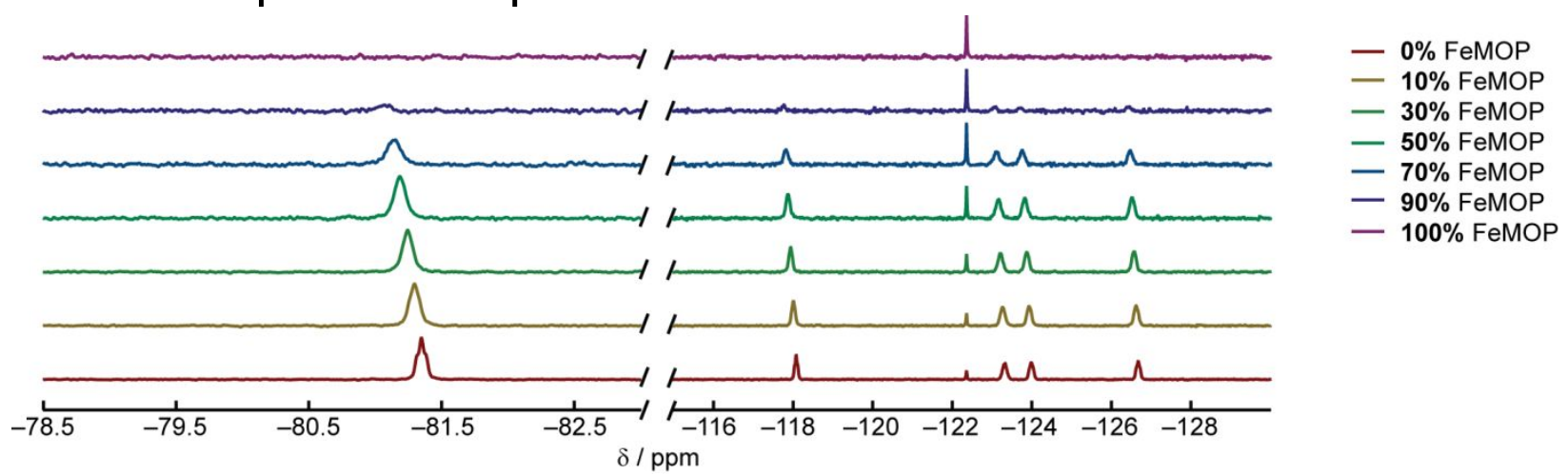

Figure S6. ${ }^{19} \mathrm{~F}$ NMR spectra of $0.5 \mathrm{~mL} \mathrm{D}_{2} \mathrm{O}$ solutions of FeMOP and PFHxA at varying ratios and a total concentration of $5.00 \mathrm{mM}$ referenced at $-122.36 \mathrm{ppm}$ using $10.0 \mathrm{mM}$ $\mathrm{NaF}$ solution in $\mathrm{D}_{2} \mathrm{O}$ for Job plot.

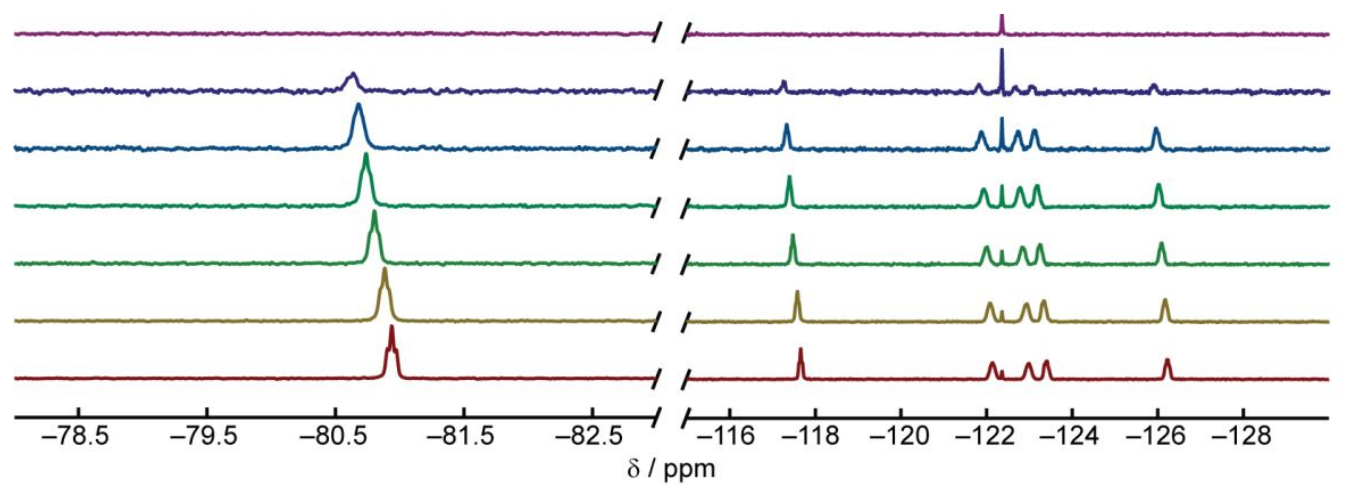

$$
\begin{aligned}
& -\mathbf{0} \% \text { FeMOP } \\
& -\quad \mathbf{1 0} \% \text { FeMOP } \\
& -\mathbf{3 0} \% \text { FeMOP } \\
& -\mathbf{5 0} \% \text { FeMOP } \\
& -\mathbf{7 0} \% \text { FeMOP } \\
& -\mathbf{9 0} \% \text { FeMOP } \\
& -\mathbf{1 0 0} \% \text { FeMOP }
\end{aligned}
$$

Figure S7. ${ }^{19} \mathrm{~F}$ NMR spectra of $0.5 \mathrm{~mL} \mathrm{D}_{2} \mathrm{O}$ solutions of FeMOP and PFHpA at varying ratios and a total concentration of $5.00 \mathrm{mM}$ referenced at $-122.36 \mathrm{ppm}$ using $10.0 \mathrm{mM}$ $\mathrm{NaF}$ solution in $\mathrm{D}_{2} \mathrm{O}$ for Job plot. 
VII. ${ }^{19} \mathrm{~F}$ Variable Temperature (VT) NMR spectra

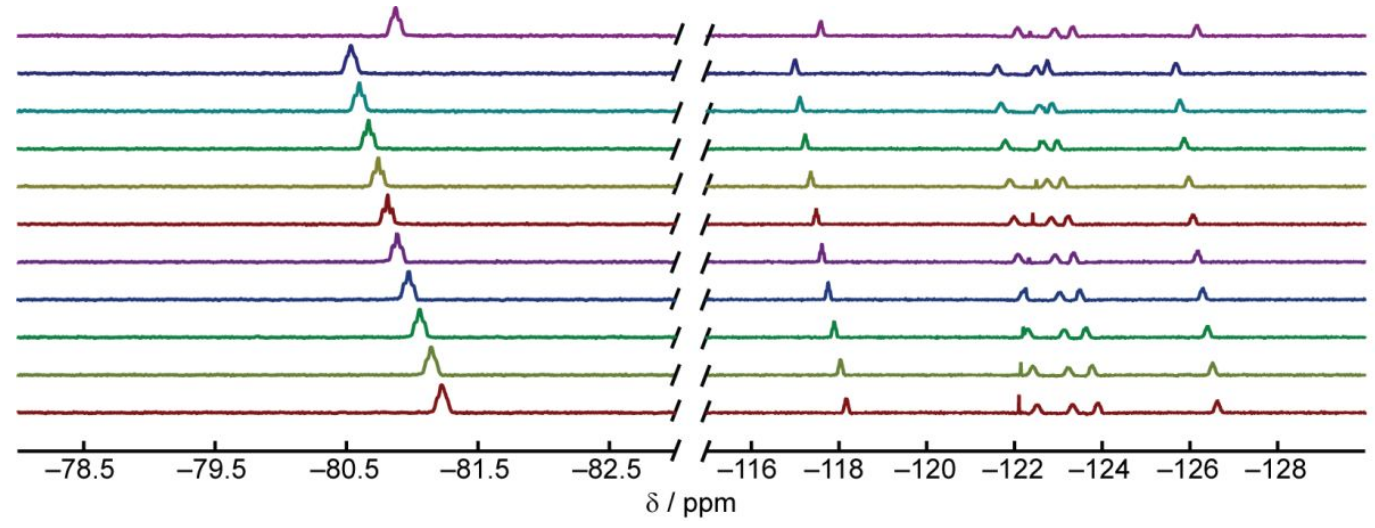

$-5^{\circ} \mathrm{C}$

$-10^{\circ} \mathrm{C}$

$-15^{\circ} \mathrm{C}$

$-20^{\circ} \mathrm{C}$

$-25^{\circ} \mathrm{C}$

$-30^{\circ} \mathrm{C}$

$-35^{\circ} \mathrm{C}$

$-40^{\circ} \mathrm{C}$

$-45^{\circ} \mathrm{C}$

$-50^{\circ} \mathrm{C}$

$-25^{\circ} \mathrm{C}$

Figure S8. ${ }^{19} \mathrm{~F}$ NMR spectra of $0.5 \mathrm{~mL} \mathrm{D}$ O solution of $5.00 \mathrm{mM} \mathrm{PFHpA}$ at $5,10,15,20$, $25,30,35,40,45^{\circ} \mathrm{C}$ then back to $25^{\circ} \mathrm{C}$ for VT NMR method.

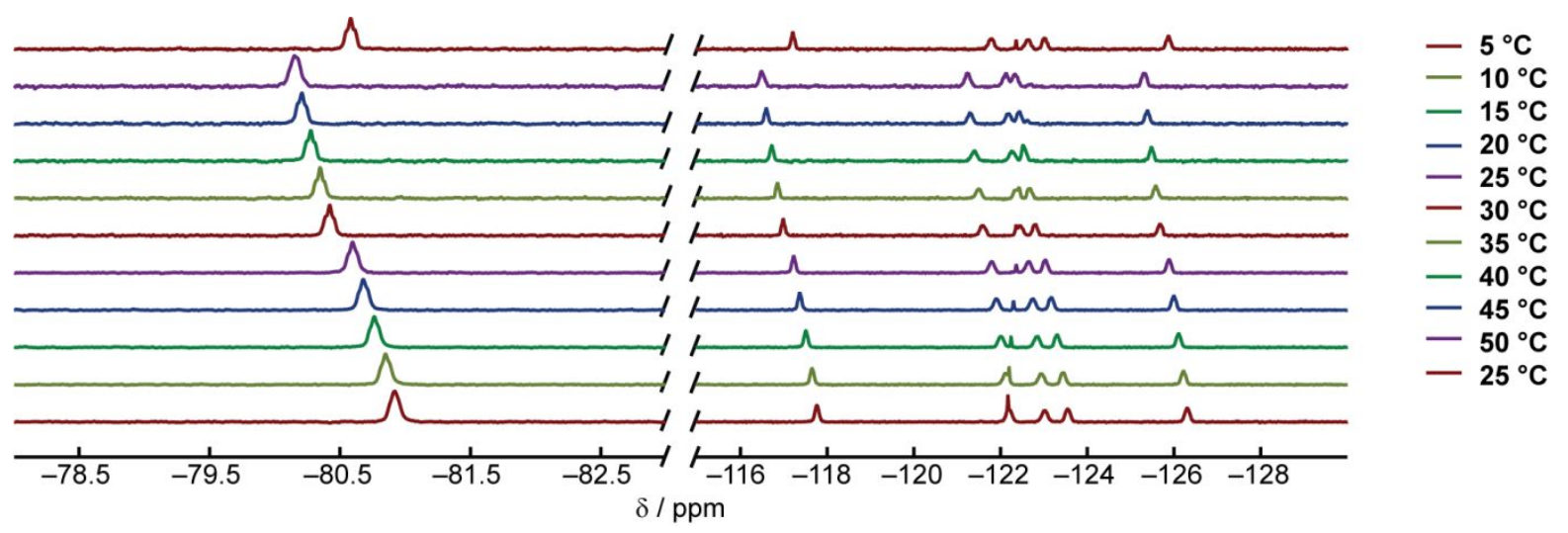

Figure S9. ${ }^{19} \mathrm{~F}$ NMR spectra of $0.5 \mathrm{~mL} \mathrm{D}_{2} \mathrm{O}$ solution of a $10.0 \mathrm{mM}$ total concentration $1: 1$ (FeMOP:PFHpA) at $5,10,15,20,25,30,35,40,45^{\circ} \mathrm{C}$ then back to $25^{\circ} \mathrm{C}$ for VT NMR method. 
Table S4. Summary of fit parameters for the VT NMR modeling of FeMOP association with PFHpA

\begin{tabular}{|c|c|c|c|c|c|c|c|c|}
\hline $\begin{array}{c}\text { Peak/R } \\
\text { un }\end{array}$ & $\mathrm{A} 1^{a}$ & $\mathrm{~A} 2^{a}$ & $x 0^{a}$ & $\mathrm{dx}^{a}$ & $\mathrm{R}_{\mathrm{adj}}{ }^{2}$ & $\mathrm{~T}_{1 / 2}, K$ & $\begin{array}{c}\Delta \mathrm{H}, \\
k J \mathrm{~mol}^{-1}\end{array}$ & $\begin{array}{c}\Delta \mathrm{S}, \\
J \mathrm{~mol}^{-1} K^{-1}\end{array}$ \\
\hline $1 / 1$ & $111.85 \pm 1$ & $99.25 \pm 1$ & $\begin{array}{c}0.00332 \pm \\
0.5\end{array}$ & $\begin{array}{c}2.08 \times 10^{-5} \pm \\
52\end{array}$ & 0.90902 & 300.92 & $4.00 \times 10^{2}$ & $1.33 \times 10^{3}$ \\
\hline $2 / 1$ & $164.18 \pm 13$ & $118.14 \pm 4$ & $0.00325 \pm 3$ & $\begin{array}{c}1.09 \times 10^{-4} \pm \\
61\end{array}$ & 0.95666 & 307.88 & $7.62 \times 10^{1}$ & $2.47 \times 10^{2}$ \\
\hline $3 / 1$ & $111.82 \pm$ & $99.92 \pm$ & $0.00332 \pm$ & $2.00 \times 10^{-5} \pm$ & ก .924ก1 & 30112 & $415 \times 1 n^{2}$ & $138 \times 10^{3}$ \\
\hline
\end{tabular}




\section{VIII. ${ }^{1} \mathrm{H}$ and ${ }^{19} \mathrm{~F}$ DOSY NMR spectra}

(a)
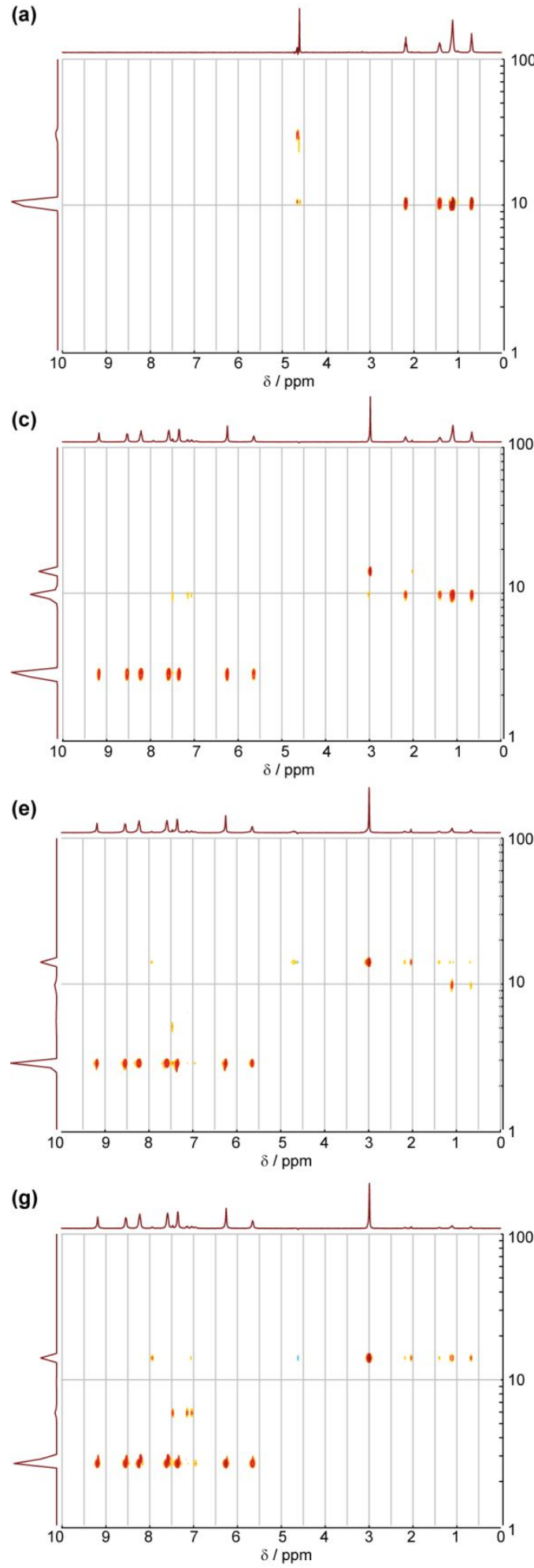
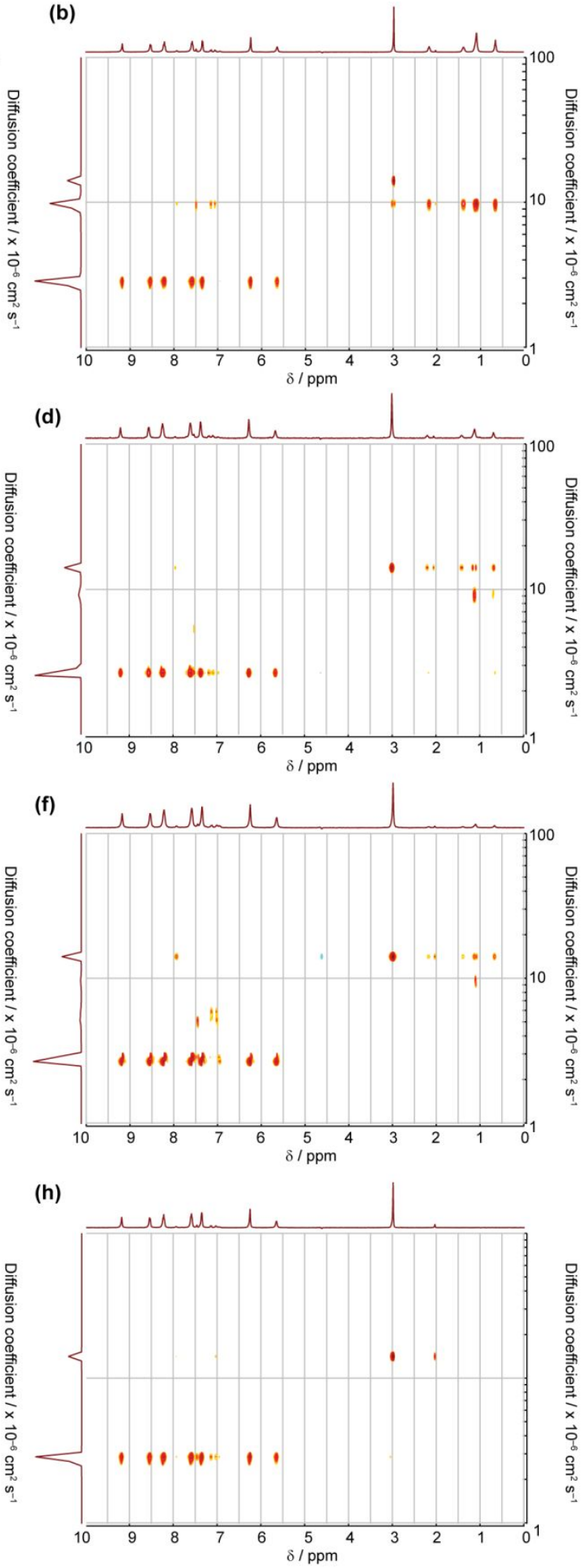

Figure S10. ${ }^{1} \mathrm{H}$ DOSY NMR spectra of (a) heptanoic acid, (b) 1:4 (FeMOP:heptanoic acid), (c) 1:3 (FeMOP:heptanoic acid), (d) 1:2 (FeMOP:heptanoic acid), (e) 1:1 
(FeMOP:heptanoic acid), (f) 2:1 (FeMOP:heptanoic acid), (g) 3:1 (FeMOP:heptanoic acid) at $2.5 \mathrm{mM}$ heptanoic acid concentration, and (h) $2.5 \mathrm{mM}$ FeMOP in $\mathrm{D}_{2} \mathrm{O}$.

(a)

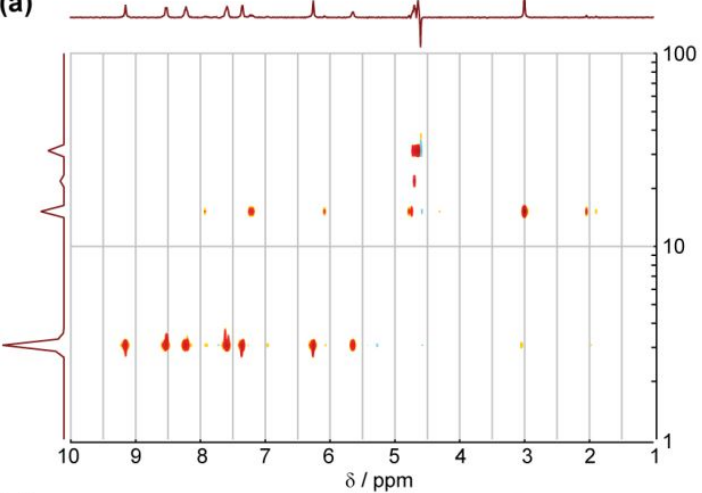

(c)

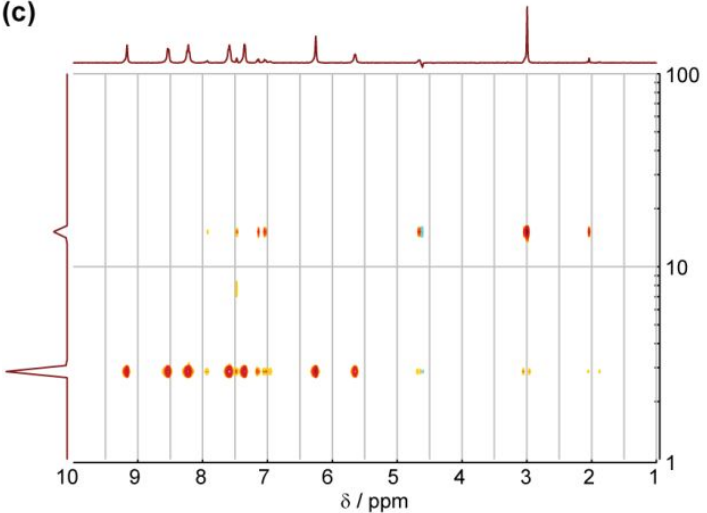

(e)

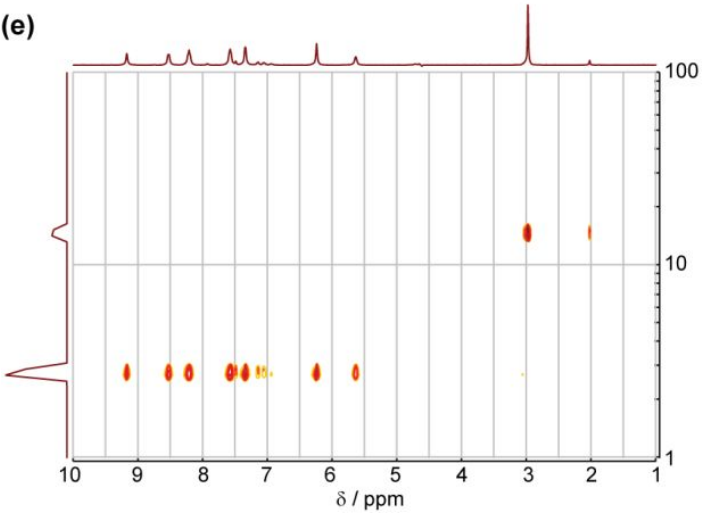

(b)

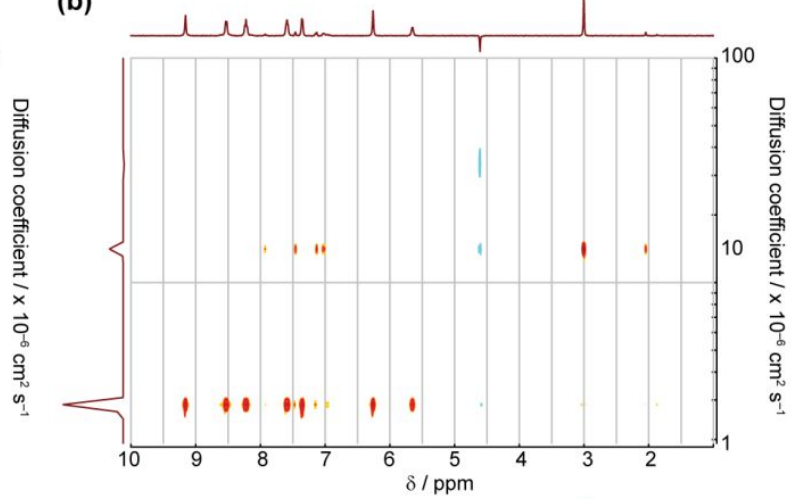

(d)
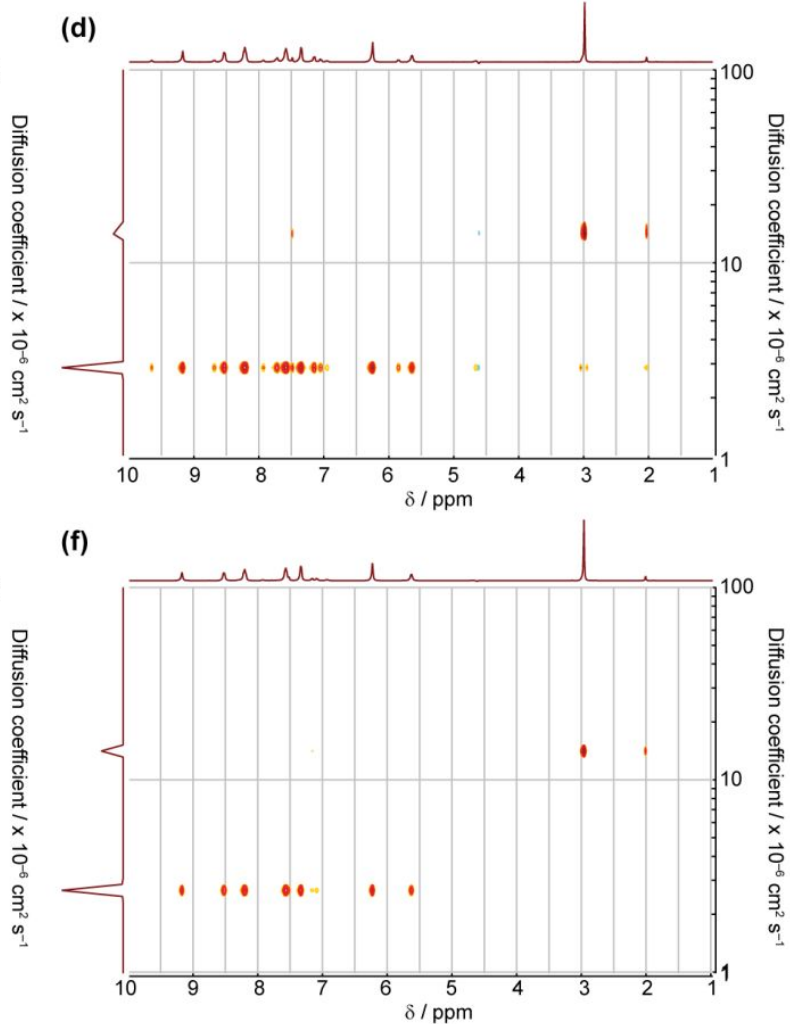

Figure S11. ${ }^{1} \mathrm{H}$ DOSY NMR spectra of (a) 1:4 (FeMOP:PFHpA), (b) 1:2 (FeMOP:PFHpA), (c) 1:1 (FeMOP:PFHpA), (d) 2:1 (FeMOP:PFHpA), (e) 3:1 (FeMOP:PFHpA), and (f) 4:1 (FeMOP:PFHpA) $\mathrm{D}_{2} \mathrm{O}$ solutions at $1.0 \mathrm{mM}$ PFHpA concentration. 

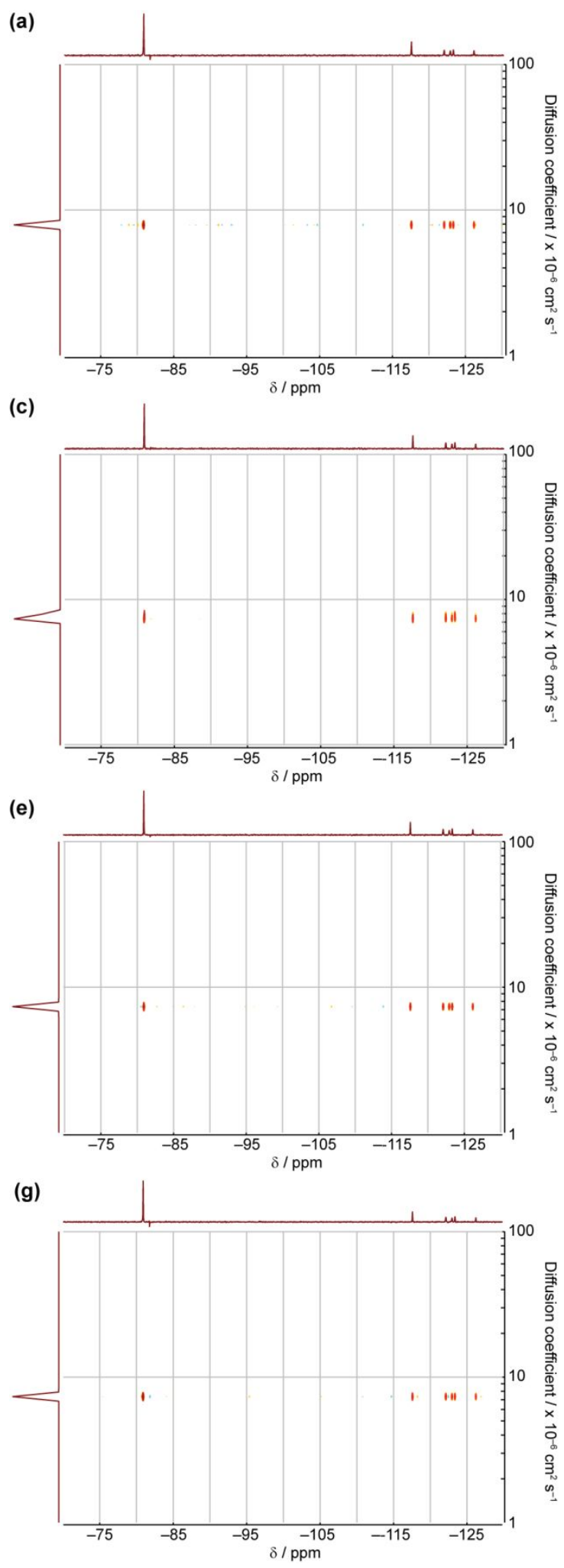

(b)

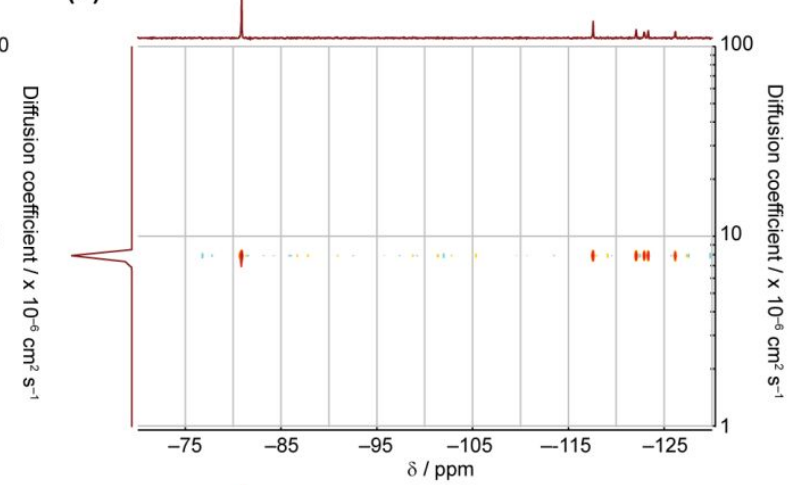

(d)

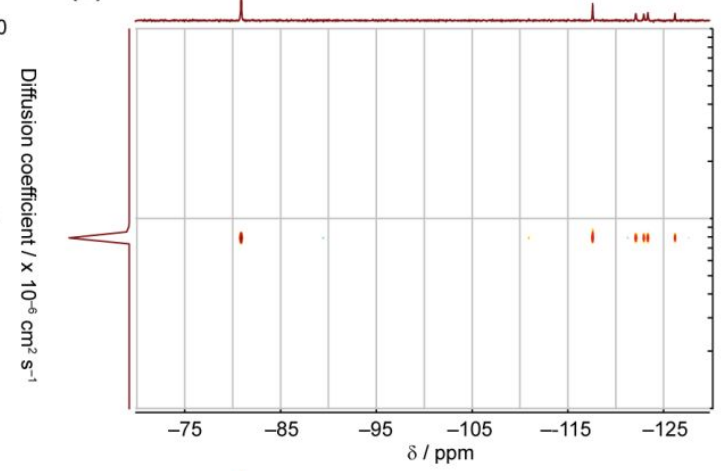

(f)

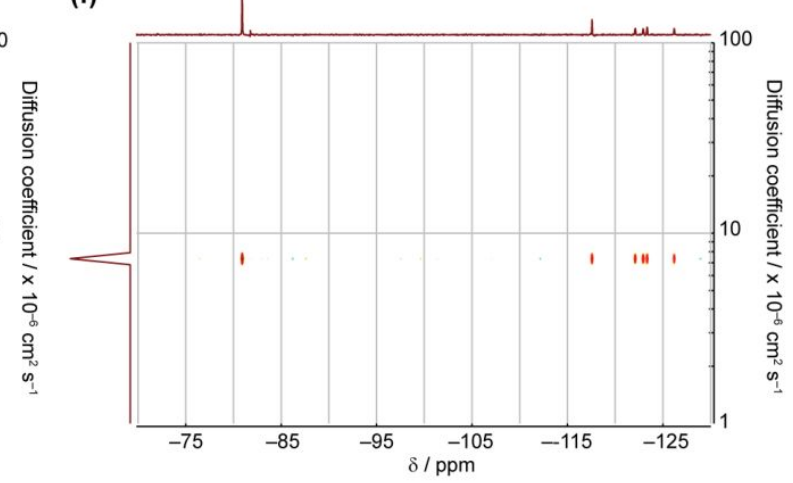

Figure S12. ${ }^{19} \mathrm{~F}$ DOSY NMR spectra of (a) PFHpA, (b) 1:4 (FeMOP:PFHpA), (c) 1:2

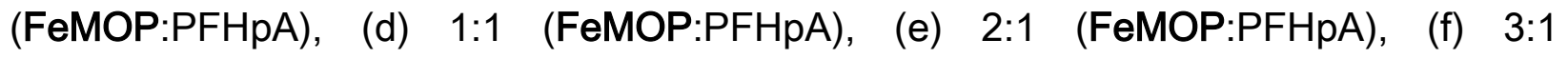


(FeMOP:PFHpA), and (g) 4:1 (FeMOP:PFHpA) $\mathrm{D}_{2} \mathrm{O}$ solutions at $1.0 \mathrm{mM}$ PFHpA concentration.

Table S5. Summary of diffusion coefficients of FeMOP when complexed with heptanoic acid and PFHpA

\begin{tabular}{cccc}
\hline Solution & \multicolumn{3}{c}{ Diffusion coefficient, $\times 10^{-6} \mathrm{~cm}^{2} \mathrm{~s}^{-1}$} \\
\hline [FeMOP]/[heptanoic acid] & FeMOP & Heptanoic acid & PFHpA \\
\cline { 2 - 4 } 0.00 & 0 & 10.5 & - \\
0.25 & 2.85 & 9.8 & - \\
0.33 & 2.86 & 9.8 & - \\
0.50 & 2.67 & 9.22 & - \\
1.00 & 2.86 & 9.73 & - \\
2.00 & 2.66 & 9.73 & - \\
3.00 & 2.67 & 9.73 & - \\
{$[$ FeMOP]/[PFHpA] } & & & 787 \\
$n \mathrm{nn}$ & $n$ & - &
\end{tabular}

\section{References}

(1) Mal, P.; Schultz, D.; Beyeh, K.; Rissanen, K.; Nitschke, J. R., An UnlockableRelockable Iron Cage by Subcomponent Self-Assembly. Angew. Chemie. Int. Ed. 2008, $47,8297-8301$. 